\title{
ASPECTOS TÉCNICOS DO IMPLANTE DE MARCA-PASSO ENDOCÁRDICO
}

\section{TECHNICAL ASPECTS OF ENDOCARDIAL PACEMAKER IMPLANTATION}

\author{
Jefferson Okamoto', Luis Daniel Silva Pilatti', Mário Augusto Cray da Costa ${ }^{1}$, \\ Alice Magro Koscianski ${ }^{1}$ \\ 'Universidade Estadual de Ponta Grossa, Departamento de Medicina, Ponta Grossa, Paraná, \\ Brasil \\ *Autor Correspondente: Endereço: Avenida Anita Garibaldi, 1555, casa 18; Telefone: (42) \\ 99804-6771; e-mail: luisdanielsp@gmail.com
}

\begin{abstract}
RESUMO
A técnica mais utilizada atualmente para a maioria dos implantes de marca-passo é a endocárdica, evitando-se os riscos da toracotomia. Dentro dessa técnica, existem diferentes métodos e variáveis para sua inserção, como as características do paciente e a técnica adotada pelo cirurgião. Dentre as principais variáveis dessa operação estão: a escolha do acesso venoso, tipo de punção e técnica de posicionamento dos eletrodos. Assim, o objetivo do presente estudo foi promover uma revisão de literatura integrativa sobre a técnica de implante de marca-passo endocárdico, com comentários baseados na experiência do autor de mais de dois mil implantes.

Descritores: Marca-passo; Procedimentos Cirúrgicos Operatórios.
\end{abstract}

\begin{abstract}
The most used technique for pacemaker implants nowadays is the endocardial, avoiding the risks of thoracotomy. Within this technique there are different methods and variables for its insertion, such as patient characteristics and the technique adopted by the surgeon. Among the main variables of this operation are the choice of venous access, type of puncture and positioning technique of the electrodes. Thus, the objective of the present study was to promote an integrative literature review on the technique of endocardial pacemaker implantation, with comments based on the author's experience of more than two thousand implants.
\end{abstract}

Keywords: Pacemaker, Artificial; Surgical Procedures, Operative. 


\section{INTRODUÇÃO}

Atualmente, a técnica mais utilizada para implante de marca-passo é a endocárdica. Porém, nas décadas de 50 e 60 , eram frequentes as grandes cirurgias com toracotomia e anestesia geral para implantação de eletrodos epimiocárdicoํ․ Em 1958, Furman, utilizando um fio no lúmen de um cateter Cournand, conseguiu, através da veia jugular externa, o acesso ao ventrículo direito de um cão, verificando que o endocárdio constituía-se em ótima zona para estimulação, além de apresentar fácil acesso para a colocação de um eletrodo. ${ }^{2}$ Menos de uma década após, um sistema endocárdico totalmente implantável era introduzido comercialmente por Chardak e pela empresa Medtronic, dando início à era dos implantes endocárdicos ${ }^{3}$. Assim, com o tempo, a técnica epimiocárdica perdeu espaço e, atualmente, é utilizada somente em situações específicas.

Quadro 1: Indicações das técnicas de implante de marcapasso.

\begin{tabular}{|l|l|}
\hline Técnica & Indicações e diferenciais \\
\hline Endocárdica & $\begin{array}{l}\text { Deve ser preferível, sempre que possível. } \\
\text { Prioritária em idosos, pneumopatas } \\
\text { crônicos, por conta da anestesia local; } \\
\text { indicada em pacientes obesos pela } \\
\text { facilidade de abordagem da via venosa } \\
\text { evitando toracotomia. }\end{array}$ \\
\hline Epimiocárdica & $\begin{array}{l}\text { Uso prioritário e indiscutível em } \\
\text { pacientes portadores de prótese de } \\
\text { tricúspide, endocardite e cardiopatias } \\
\text { congênitas }{ }^{4} \text {. Usada em crianças de } \\
\text { baixo peso, em pacientes com ausência } \\
\text { de possibilidade de acesso venoso, } \\
\text { anomalias anatômicas, quando outros } \\
\text { procedimentos cirúrgicos no coração } \\
\text { estão sendo realizados, em caso de } \\
\text { tratamento de complicações do método } \\
\text { endocárdico e, em situações em que não } \\
\text { se dispõe de fluoroscopia. }\end{array}$ \\
\hline
\end{tabular}

Fonte: o Autor

\section{MÉTODO}

Pesquisa integrativa na literatura, na base de dados MEDLINE, LILACS e SciELO. Foi realizado levantamento de artigos completos publicados em línguas inglesa ou portuguesa de 1957 até o ano de 2018. O período de busca foi de março de 2016 até maio de 2018, sendo utilizados para busca dos artigos, os seguintes descritores e suas combinações em ambos os idiomas: "Marca-Passo Artificial" e "técnica cirúrgica". A busca resultou em 2106 artigos nas três bases de dados, sendo excluídos artigos que abordavam marca-passos epimiocárdicos, válvulas cardíacas, ressincronização, desfibrilador, resultando, assim, 93 artigos dos quais foram selecionados manualmente 12 para a confecção dessa revisão. Publicações adicionais foram identificadas utilizando-se a lista de referências dos manuscritos selecionados.

\section{REVISÃO \\ TÉCNICA DE IMPLANTE ENDOCÁRDICO}

\section{Sala cirúrgica e materiais}

Todos os implantes de marca-passo devem ser realizados em ambiente limpo e asséptico, no bloco cirúrgico ou sala de hemodinâmica. As salas devem ser equipadas com desfibrilador, monitor multiparamétrico com pelo menos monitor de ritmo cardíaco e oximetria, fluoroscopia ou intensificador de imagens com circuito interno de televisão. Possuindo variedade de geradores de câmara simples e dupla, eletrodos de fixação ativa e passiva, eletrodos atriais e ventriculares e várias bainhas introdutoras de diversos diâmetros, programador e cabos elétricos estéreis.

\section{Orientação do paciente}

Uma vez checados os materiais, o paciente deve ser orientado sobre todos os passos do implante e o que ele poderá sentir durante o procedimento. Devese explicar sobre a necessidade de manter-se parado e cooperativo durante o ato cirúrgico. Um ansiolítico por via oral pode ser recomendável em pacientes muito ansiosos. Em raras situações, em pacientes com problemas mentais, doença de Alzheimer ou muito ansiosos, pode ser necessária uma sedação mais profunda com o auxílio da equipe de anestesia.

\section{Preparo do paciente}

O paciente deve ser posicionado em decúbito dorsal no momento em que se realiza o acesso venoso periférico preferencialmente no braço contralateral ao lado do implante, que deve ser mantido com soro fisiológico em gotejamento muito lento, somente para manutenção do acesso. Lembrar que muitos pacientes 
têm disfunção ventricular esquerda e não suportam sobrecarga volumétrica. Monitorizar com oximetria de pulso, cardioscópio e pressão não invasiva com manguito em braço direito, salvo em caso de obstrução arterial deste membro. A região peitoral deve ser rigorosamente limpa para evitar infecções. Para tanto, deve-se realizar tricotomia seguida de lavagem com solução degermante de clorexidine ou Polivinil Pirrolidona Iodo (PVPI), e seca com compressa estéril e realização de antissepsia com clorexidine a $0,2 \%$ ou PVPI tópico.

A antibioticoprofilaxia no implante do marca-passo pode ser realizada com dois fármacos, dependendo da disponibilidade do serviço. Na chegada do paciente ao centro cirúrgico, pode-se administrar cefuroxima 1,5 $\mathrm{mg}$ via endovenosa $(\mathrm{EV})$ como dose de indução, e $750 \mathrm{mg}$ após 12 horas de pós-operatório; ou uma dose única de cefazolina $2 \mathrm{~g}$ via $\mathrm{EV}$ antes do procedimento $^{5}$. Caso o paciente esteja internado há mais de 48 horas ou ainda, seja alérgico à penicilina, recomenda-se a administração de vancomicina ${ }^{6,7}$

Os autores utilizam administração de cefazolina $1 \mathrm{~g}$ para pacientes com até $70 \mathrm{Kg}$ de peso corporal e $2 \mathrm{~g}$ para pacientes maiores antes da cirurgia e $1 \mathrm{~g}$ a cada 8 horas por 24 horas no período pós-operatório, porém não é recomendação absoluta a antibioticoterapia profilática.

\section{Vias de acesso}

O posicionamento dos campos cirúrgicos se dá no local do acesso venoso.

Existem diversos locais de acesso

Quadro 2: Vias de acesso da técnica de implante endocárdico

\begin{tabular}{|c|c|c|}
\hline $\begin{array}{l}\text { Vias de acesso } \\
\text { endocárdicas }\end{array}$ & Diferenciais & Método \\
\hline Veia cefálica & $\begin{array}{l}\text { Acesso fácil e grande viabilidade de uso }{ }^{4} \text {. } \\
\text { Entretanto, em alguns casos, ela é muito fina }{ }^{4,6} \text {. } \\
\text { Precisa de mais habilidade por parte do cirurgião }{ }^{8} \text {. } \\
\text { O risco de pneumotórax é }<0,1 \% \text {, necessita de } \\
\text { baixa quantidade de fluoroscopia. Entretanto, } \\
\text { por essa via, a passagem de múltiplos cateteres e } \\
\text { extração de eletrodos é dificultada. }\end{array}$ & $\begin{array}{l}\text { A veia cefálica está no sulco deltopeitoral, mais } \\
\text { próxima da borda do músculo grande peitoral. Após } \\
\text { a dissecação da veia, faz-se uma ligadura com o fio } \\
\text { inabsorvível distalmente e outra falsa ligadura mais } \\
\text { proximal para permitir a exposição }{ }^{4} \text {. }\end{array}$ \\
\hline $\begin{array}{l}\text { Veias } \\
\text { Jugulares } \\
\text { Internas ou } \\
\text { Externas }\end{array}$ & $\begin{array}{l}\text { Necessita maior precisão cirúrgica; é necessário } \\
\text { fazer uma conexão (túnel) até loja no tórax }{ }^{4} \text {. }\end{array}$ & $\begin{array}{l}\text { Coloca-se o paciente na posição de Tredelenburg } \\
\left(10^{\circ}-15^{\circ} \text { de elevação), e virando seu rosto para o lado }\right. \\
\text { contralateral. Identifica-se o ponto de inserção na } \\
\text { borda posterior do ECM, no nível superior de onde a } \\
\text { veia jugular externa cruza o músculo } 9\end{array}$ \\
\hline Subclávia & $\begin{array}{l}\text { Não requer tanta habilidade quanto a veia } \\
\text { cefálica, entretanto, o risco de pneumotórax é de } \\
1-2 \% \text {. Requer baixa quantidade de fluoroscopia, } \\
\text { permitindo o acesso mais fácil de múltiplos } \\
\text { eletrodos e sua extração }{ }^{4} \text {. } \\
\text { É contraindicada em casos de anomalias } \\
\text { anatômicas, trauma de clavícula, parte anterior da } \\
\text { costela e coagulopatias. Como contraindicação } \\
\text { relativa está o DPOC. }{ }^{9}\end{array}$ & $\begin{array}{l}\text { O ponto de referência para a punção é a união do } \\
\text { terço médio ao terço medial da clavícula. Palpando- } \\
\text { se com o indicador este ponto, pode-se perceber sob } \\
\text { a clavícula o ponto de cruzamento com a costela, } \\
\text { este é o local adequado para a punção com a agulha } \\
\text { em } 45 \text { graus com a pele e a ponta direcionada para a } \\
\text { fúrcula esternal. Uma vez que o músculo é perfurado, } \\
\text { avança-se a agulha de lateral pra medial }{ }^{8} \text {. A agulha } \\
\text { deve "deslizar" por baixo da clavícula para diminuir } \\
\text { o risco de pneumotórax. }\end{array}$ \\
\hline
\end{tabular}


conclusão

\begin{tabular}{|l|l|l|}
\hline $\begin{array}{l}\text { Vias de acesso } \\
\text { endocárdicas }\end{array}$ & Diferenciais & Método \\
\hline \multirow{5}{*}{ Femoral } & $\begin{array}{l}\text { É indicada em emergências durante ressuscitação } \\
\text { cardiopulmonar, provendo uma rota para } \\
\text { administração de medicamentos, acesso de } \\
\text { urgência em hemodiálise, pacientes com overdose. } \\
\text { É contraindicada em distúrbios de coagulação, } \\
\text { ausência de artéria femoral palpável, histórico } \\
\text { de vasculite e cateter prévio de longa data. A } \\
\text { vantagem da via é que o risco de pneumotórax e } \\
\text { hemotórax é mínimo. A desvantagem desse local } \\
\text { é que ele é frequentemente contaminado devido à } \\
\text { proximidade com a área perineal }\end{array}$ & $\begin{array}{l}\text { Para a punção da veia femoral, palpa-se a artéria } \\
\text { femoral e desvia-se dela, lateralmente, em torno de 1 } \\
\text { cm. }\end{array}$ \\
\hline Axilar & $\begin{array}{l}\text { O curso da veia axilar é mais variável. Entretanto, } \\
\text { é um método seguro, permite acesso rápido em } \\
\text { todos os pacientes }{ }^{10} \text {. }\end{array}$ & $\begin{array}{l}\text { Feita sobre a segunda costela, geralmente um } \\
\text { venograma é feito para orientar a punção. Utiliza-se } \\
\text { também fluoroscopia para marcar o local de punção. } \\
\text { Cuidado para não atravessar a } 1^{\mathrm{a}} \text { costela }\end{array}$ \\
\hline
\end{tabular}

Fonte: o Autor

A dissecção da veia cefálica e a punção da veia subclávia são os acessos mais utilizados. A veia cefálica representa, em grande parte das vezes, uma opção com ampla viabilidade de uso, apesar de que a passagem de múltiplos cateteres e a retirada de eletrodos é dificultada por essa via, uma vez que ela pode ser muito fina $^{4,6,8}$. A punção da veia subclávia, por sua vez, não requer tanta habilidade por parte do cirurgião como a da veia cefálica, além de permitir mais facilmente o acesso e extração de múltiplos eletrodos ${ }^{4}$. Entretanto, esta não é recomendada em casos de doença pulmonar obstrutiva crônica (DPOC) grave, uma vez que o risco de desenvolvimento de pneumotórax é maior?.

\section{Punção de subclávia}

Pode-se escolher entre uma punção antes ou após a incisão da pele para a formação da loja. A vantagem de se puncionar antes da incisão, é que se torna desnecessária a realização uma segunda incisão no lado contralateral caso não se consiga puncionar. Além disso, ao puncionar com pele fechada, os pontos de referência anatômicos são mais facilmente identificáveis, facilitando a punção. Entretanto, nesse método, deve-se ter cautela ao fazer a incisão para não lesar os eletrodos já passados, uma vez que estão num espaço estreito.

Os autores preferem utilizar de imediato a punção da subclávia. Assim, posicionam-se os campos cirúrgicos, delimitando a área de acesso para a punção venosa profunda das veias jugulares internas e veias subclávias, bilateralmente, sendo colocado o intensificador de imagem de arco em " $\mathrm{C}$ " à direita, para acesso à subclávia esquerda, posicionando o botão anestésico no local da punção. Os autores utilizam lidocaína a $2 \%$, sem vasoconstritor, diluída em 1:1 em soro fisiológico. A literatura descreve, ainda, o uso de bupivacaína a 0,5 ou 0,75 . Primeiramente, anestesia-se a pele. Em seguida, os planos mais profundos no trajeto da punção da veia subclávia, alertando o paciente sobre a dor e desconforto que ele poderá sentir durante este ato.

Em seguida, o cirurgião palpa o espaço de cruzamento entre a clavícula e a primeira costela, realizando a punção venosa profunda com agulha e seringa próprias, num ângulo de 45 graus em relação à pele com a ponta da agulha direcionada para a fúrcula esternal.

Uma vez que o músculo é perfurado, avança-se a agulha de lateral para medial ${ }^{8}$. A ponta da agulha deve ser direcionada para a fúrcula esternal, sendo que ela deve "deslizar" por baixo da clavícula para diminuir o risco de pneumotórax. Durante este processo, a inserção da agulha deve ser de maneira lenta, sempre mantendo a aspiração do êmbolo, a fim de haver uma pressão negativa no interior da seringa. Ocorre a interrupção do movimento se houver a aspiração de sangue venoso. Neste momento, a mesma é retirada para ser passado o fio guia. Explica-se ao paciente que 
ele poderá sentir um profundo desconforto devido à passagem do guia e, posteriormente, do dilatador e bainha introdutora, lembrando que é contraindicado o uso da bainha introdutora em suspeita de obstrução da veia subclávia e portadores de doenças pulmonares crônicas graves.

Realiza-se a primeira tomada instantânea de imagem para certificar-se de que o guia está em posição. A imagem é utilizada para corrigir posição da tela colocando a parte cefálica da imagem na posição vertical mais alta da imagem.

\section{Passagem do segundo fio guia por uma segunda punção}

Se a punção for fácil, após a passagem do primeiro fio faz-se uma incisão de 3 a $5 \mathrm{~mm}$ para então se realizar a segunda punção com a mesma técnica, passando o segundo fio guia. A vantagem de se ter duas punções é criar uma distância entre os eletrodos para evitar que a manipulação de um eletrodo desloque o outro.

\section{Passagem dos dois fios guias pela mesma punção}

Caso haja dificuldade para a punção, os autores sugerem que seja passado o dilatador, depois a primeira bainha 7F. Em seguida, retira-se o dilatador mantendo-se o primeiro fio guia e passa-se o segundo fio guia por dentro desta bainha. Retira-se então a bainha íntegra e tendo-se os dois fios guias em posição, passam-se separadamente duas bainhas $7 \mathrm{~F}$, uma em cada fio guia. O desafio desse método com punção única surge à medida que ao passar dois fios guias pela mesma bainha eles ficam muito próximos um do outro, podendo haver contato e, eventualmente, um pode acabar por empurrar ou tracionar o outro eletrodo.

\section{Passagem dos eletrodos}

Após a passagem da primeira bainha, passa-se o primeiro eletrodo, deixando-o na altura do átrio; depois, passa-se a segunda bainha e o segundo eletrodo, que também é conduzido até a altura do átrio. Deve-se minimizar o uso de radiação nesta etapa, que é usado somente após a introdução dos dois eletrodos para fazer a segunda tomada de raios-X rápida para se certificar da posição dos eletrodos. O movimento de eletrodos deve ser suave e a introdução suficiente apenas para que os eletrodos alcancem o átrio direito.
Após a introdução dos eletrodos no átrio, procede-se ao posicionamento e fixação do eletrodo atrial e ventricular nesta sequência.

Um cuidado adicional é que após a retirada do fio guia com dilatador, deve ser realizada a oclusão da bainha com o dedo para evitar a entrada de ar ou sangramento até que se introduza o eletrodo.

Pode escolher-se, ainda, entre fazer a segunda punção antes ou após a colocação do primeiro eletrodo. Os autores fazem em uma primeira etapa a passagem dos fios guias das bainhas, depois introduzem os eletrodos até os átrios e, somente após esse procedimento, fazem o posicionamento dos eletrodos. Isso faz com que esta primeira etapa da cirurgia seja muito rápida e com mínima exposição de raios X. Além disso, evita-se que um eletrodo já posicionado possa ser deslocado durante as manobras de colocação de uma nova bainha.

\section{Posicionamento dos eletrodos}

Após a introdução de ambos os eletrodos, inicia-se a utilização do fluoroscópio contínuo para posicionamento dos eletrodos. $\mathrm{O}$ acionamento dos raios $\mathrm{X}$ deve ser feito apenas durante os movimentos dos eletrodos para evitar exposição desnecessária de radiação da equipe e do paciente.

No implante de marca-passo dupla-câmara, os cabos-eletrodos devem ser posicionados, obviamente, um de cada vez. Muitos cirurgiões preferem iniciar pelo ventricular e introduzir o atrial posteriormente, afirmando diminuir o risco da ocorrência de um bloqueio átrio-ventricular total (BAVT) ou assistolia prolongada ${ }^{6}$. Outros alegam que devido ao maior número de manobras para cabo-eletrodo atrial o mesmo deve ser colocado primeiro. Os autores preferem a introdução do cabo-eletrodo atrial primeiramente pelo risco de deslocar o eletrodo ventricular nas manobras de posicionamento do atrial. Quando o eletrodo ventricular é posicionado primeiro, o paciente pode desenvolver dependência do estímulo e, como a guia atrial tem formato de “j”, pode deslocar o eletrodo ventricular ao ser manipulada, causando uma situação de emergência.

\section{Posicionamento do eletrodo atrial}

Após a passagem dos dois eletrodos, procede-se ao posicionamento dos mesmos começando pelo eletrodo atrial. Troca-se a guia reta por uma guia em “j” no eletrodo atrial deixando-se o eletrodo na parte inferior do átrio direito e, posterior tração do mesmo, 
até que a ponta enrosque em uma trabécula, o que é observado pelo aumento da abertura do " j". Neste instante, faz-se a fixação do eletrodo atrial ativo. Depois disso, realiza-se o teste com leve tração e introdução do eletrodo com a guia para certificar-se que está fortemente fixado. Primeiro tenta-se o posicionamento com a ponta direcionada medialmente, se não houver sucesso após duas ou três tentativas, o movimento é repetido com a ponta direcionada para a parede lateral.

Os eletrodos podem ser tanto de fixação ativa como passiva. Os de fixação passiva são os mais utilizados na estimulação do ventrículo direito. Eles apresentam garras na ponta, funcionando como uma âncora que, após determinado tempo, fixa-se na parede do miocárdio por fibrose. Já o de fixação ativa apresenta um parafuso retrátil na ponta, que é liberado quando o eletrodo está em posição, sendo recomendado em casos de cardiomegalias e pacientes com corpo trabeculado escasso. Além disso, a fixação ativa é a mais utilizada em pacientes jovens, uma vez que há uma maior chance do marca-passo precisar ser substituído ${ }^{6,11}$.

Depois da fixação do eletrodo atrial o técnico procede às aferições elétricas, com medidas de onda "p", impedância e limiar de estimulação.

\section{Posicionamento do eletrodo ventricular}

Durante a realização das medidas do eletrodo atrial, para reduzir o tempo cirúrgico, o cirurgião pode proceder ao posicionamento do eletrodo ventricular. Introduz-se um pouco o eletrodo com a guia reta e se o mesmo migrar para cava inferior, retira-se a guia e faz-se uma curvatura na guia que é adaptada ao tamanho da área cardíaca de forma que a curvatura possa ficar no átrio direito $(\mathrm{AD})$ e a ponta do eletrodo na ponta do ventrículo direito (VD). Deve-se sempre fazer a curvatura com a mão limpa de sangue e úmida com soro e com o auxílio de uma pinça hemostática. A presença de sangue na guia pode dificultar o seu movimento por dentro do eletrodo. Muitas vezes, o eletrodo não entra na primeira tentativa. Neste caso, se a ponta estiver fixa em algum ponto $\mathrm{AD}$, pode-se progredir o eletrodo introduzindo-o de forma que o mesmo faça uma curvatura para dentro do VD. Devemos progredir bastante o eletrodo para que haja grande extensão do mesmo em VD. Nesta situação, observa-se a ponta do eletrodo fixo no átrio e uma curvatura do mesmo atravessando a válvula tricúspide para dentro do ventrículo.
Após este processo, retira-se cuidadosamente a guia e, em seguida, passa-se uma guia reta ainda não usada, tracionando o eletrodo, de forma que a ponta da guia reta fique dentro da curvatura na porção interna de VD. O eletrodo deve ser tracionado até que sua ponta faça um movimento de chicote e entre no VD. A seguir, é só o conduzir até a ponta, fazer a fixação e proceder às medidas de sensibilidade (onda $\mathrm{R}$ ), impedância e limiar, realizadas pelo técnico. Para determinar o limiar - o mínimo de corrente necessária para ocorrer captura - aumenta-se ao máximo a corrente elétrica e é aplicada uma frequência entre $60-80 \mathrm{bpm}$ ou uma acima do paciente no momento do implante, então a corrente é diminuída até perda de captura. O ideal é um valor menor que $1 \mathrm{~mA}$ que é multiplicado por 2-2,5. Se o valor for acima de 5-6 mA, o cateter deve ser reposicionado ${ }^{12}$. A impedância deve estar entre 400 e 1000 ohms, e a sensibilidade da onda P deve ser $\geq$ $1.5 \mathrm{mv}, \mathrm{e} \geq 5.0 \mathrm{mv}$ na onda $\mathrm{R}^{11}$.

\section{Confecção da loja do marca-passo}

Enquanto o técnico faz as medidas, o cirurgião realiza a anestesia na região da loja e procede à confecção da bolsa do gerador. A incisão começa pelo orifício de punção e segue em direção lateral e levemente inferior. Dissecam-se as partes moles e abre-se a fáscia do peitoral maior, que é descolada, confeccionando-se a loja subfascial. O gerador ainda pode ser implantado acima da fáscia do peitoral maior, especialmente naqueles pacientes com farto tecido subcutâneo, sendo que a implantação nessa região é mais fácil e menos dolorosa ${ }^{8}$. Por fim, verifica-se a hemostasia do paciente.

\section{Finalizando o procedimento}

Se as medidas de ambos os eletrodos estiverem dentro dos parâmetros ótimos para estimulação, retiram-se os guias sob radioscopia e observam-se as curvaturas, as quais devem ser confortáveis, evitando que os eletrodos fiquem esticados. Posteriormente, fixam-se os mesmos com fio 2-0. A seguir, conectam-se os eletrodos ao gerador, com o cuidado de introduzir completamente o eletrodo no gerador, a ponta do eletrodo pode ser observada cruzando o parafuso. Depois, coloca-se o gerador na loja com os fios enrolados sob o aparelho. É importante lembrar que ao introduzir o gerador na loja não se deve dobrar excessivamente o cabo próximo a ele, sob pena de causar danos ao isolamento 
do cabo-eletrodo. Deve-se, ainda, fixar o gerador na loja evitando que o mesmo se desloque (Síndrome de Twiddler). Sutura-se a fáscia com a ponta agulhada do fio utilizado para fixar os eletrodos no músculo peitoral. Essa sutura deve ser feita em chuleio até a extremidade da incisão e voltada em chuleio pelo subcutâneo até encontrar a outra ponta, dando-se, enfim, o nó. A seguir, faz-se a sutura da pele com Vycril 3-0 incolor em sutura intradérmica. Por último, realiza-se o curativo, deixando-se um peso de $1 \mathrm{Kg}$ sobre ele por 6 horas no pós-operatório para evitar hematomas.

\section{CONSIDERAÇÕES FINAIS}

No implante de um marca-passo cardíaco endocárdico, existem diversas variáveis para escolha do método e da via de acesso, dependendo do perfil do paciente, suas patologias concomitantes, da preferência e experiência do cirurgião. Essas variáveis devem ser analisadas e escolhidas visando reduzir a chance de complicações. Uma padronização de técnica pode minimizar o tempo de cirurgia e o tempo de exposição à radiação.

\section{REFERÊNCIAS}

1. Weirich WL, Gott VL, Lillehei CW. The treatment of complete heart block by the combined use of myocardial electrode and an artificial pacemaker. Surg forum. 1957; 8:360-3.

2. Furman S, Robinson G. Stimulation of the ventricular endocardial surface in control of complete heart block. Ann Surg. 1959; 150:841-5.

3. Senning A. Problems in the use of pacemakers. J Cardiovasc Surg. 1964; 5:651-6.

4. Gauch PRA, Andrade JCS. Técnica de implante de marca-passo endocárdico. Rev. bras. marca-passo e arritmia. 1989; 2 (2):65-76.

5. Dias MBS, Corradi MFDB, Mattar Júnior J. Protocolo de antibioticoprofilaxia no paciente cirúrgico. Hospital Sírio-Libanês, 2015

6. Bronchtein S. Implante de marca-passo cardíaco definitivo. Aspectos cirúrgicos. Rev. SOCERJ. 2002; 15 (2): 102-112.

7. Bratzler DW, Dellinger EP, Olsen KM, Perl TM, Auwaerter PG, Bolon MK et al. Clinical practice guidelines for antimicrobial prophylaxis in surgery. Surgical Infections. 2013; 14(1):73-156.
8. Rajappan K. Permanent pacemaker implantation technique: part I. Heart. 2009; 95:259-264.

9. Roe EJ, Turner-Lawrence D. Central Venous Access via Subclavian Approach to Subclavian Vein Technique. Medscape. 2017.

10. Burri H, Sunthorn H, Dorsaz PA, Shah D. Prospective study of axillary vein puncture with or without contrast venography for pacemaker and defibrillator lead implantation. Pacing Clin Electrophysiol. 2005; 28(1): 280-3.

11. Rajappan K. Permanent pacemaker implantation technique: part II. Heart. 2009; 95(4):334-42.

12. Harrigan RA, Chan TC, Moonblatt S, Vilke GM, Ufberg JW. Temporary transvenous pacemaker placement in the Emergency Department. J Emerg Med. 2007; 32(1):105-11. 